\title{
Investigación en educación médica - IEM en la Facultad de Medicina de San Fernando
}

Pedro Mendoza

Universidad Nacional Mayor de San Marcos

Objetivos: Identificar investigadores en educación médica (IEM) o investigación educacional en salud; identificar qué líneas se viene investigando, y estrategias metodológicas principales.

Diseño: Transversal.

Institución: Universidad Nacional Mayor de San Marcos.

Participantes: Investigadores de Departamentos e Institutos de la Facultad.

Intervenciones: Estudio bibliométrico.

Principales medidas de resultados: Unidades bibliográficas.

Resultados: En 120 unidades bibliográficas identificadas en el periodo 2005 a 2010, las principales líneas de investigación en educación médica en la Facultad fueron: Métodos de Enseñanza-Aprendizaje; Formación en Investigación; Ética; Características de los Estudiantes; Admisión/Egreso; e Investigación Curricular. No hubo investigación en gestión educativa. Solo $25 \%$ de la producción era tipo artículo, $75 \%$ resúmenes. Las principales unidades que investigaron en educación médica fueron los DA de Ciencias Dinámicas, Medicina Preventiva y Enfermeria, y el Instituto de Etica. La investigación fue efectuada en $80 \%$ por equipos, en su mayoría de 3 a 6 docentes. Hubo un importante aporte de los estudiantes a través de la Sociedad Científica de San Fernando. La metodología empleada generalmente fue el diseño descriptivo y estudios de caso.

Conclusiones: Se debe articular la experiencia y capacidad de investigación en educación médica de la Facultad, con la agenda de prioridades de investigación en recursos humanos en salud establecida por el Ministerio de Salud.

Palabras clave: Investigación educacional, educación médica.

\section{Conocimientos y actitudes hacia los derechos del paciente en estudiantes de Enfermería de la Facultad de Medicina de la UNMSM}

Carmen Del Carmen, Juana Durand, Tula Espinoza, Luisa Rivas, Yissella Acuache, Luzmila Figueroa, Roberto Paredes

EAP de Enfermeria, Facultad de Medicina, UNMSM

Objetivos: Identificar los conocimientos sobre los derechos del paciente y las actitudes hacia los derechos del paciente en estudiantes de Enfermería de la FM de la UNMSM, e identificar áreas críticas en la formación de dichos estudiantes relacionadas con los derechos de los pacientes.

Diseño: Estudio cuanticualitativo, método descriptivo simple de corte transversal.

Institución: EAP de Enfermería, Facultad de Medicina, UNMSM.

Participantes: Estudiantes de Enfermería.

Intervenciones: Encuesta a 213 estudiantes de Enfermería, por muestreo probabilístico, para la aplicación del cuestionario y escala de Lickert modificada, previa validez y confiabilidad estadística.

Principales medidas de resultados: Conocimientos, actitudes y áreas críticas en la formación sobre derechos del paciente, en estudiantes de Enfermería.

Resultados: No conocía los derechos del paciente el 64\%, actitud medianamente favorable en $62 \%$, desfavorable en $31 \%$ y favorable en $7 \%$. Áreas críticas: conceptualización de los derechos del paciente, devenir histórico de los derechos humanos, derecho a la dignidad, a la comunicación y al consentimiento informado.

Conclusiones: La mayoría de estudiantes no conocía los derechos del paciente; la actitud de la mayoria fue medianamente favorable y las áreas críticas en la formación incluyeron la conceptualización sobre derechos del paciente, el devenir histórico de los derechos humanos, el derecho a la dignidad, la comunicación y el consentimiento informado.

Palabras clave: Conocimientos sobre derechos del paciente, actitudes hacia los derechos del paciente, formación profesional. 